\title{
Altered integrity of corpus callosum in generalized epilepsy in relation to seizure lateralization after corpus callosotomy
}

\author{
Po Ching Chen, PhD, ${ }^{1,2}$ Steven A. Messina, MD, ${ }^{3}$ Eduardo Castillo, PhD, ${ }^{2}$ \\ James Baumgartner, MD, ${ }^{4}$ Joo Hee Seo, MD, ${ }^{1}$ Holly Skinner, DO, ${ }^{1}$ Elakkat D. Gireesh, MD, \\ and Ki Hyeong Lee, MD' \\ ${ }^{1}$ Epilepsy Center, ${ }^{2} \mathrm{MEG}$ Center, and ${ }^{4}$ Neurosurgery, Neuroscience Institute, Florida Hospital, AdventHealth, Orlando, Florida; \\ and ${ }^{3}$ Department of Radiology, Mayo Clinic, Rochester, Minnesota
}

\begin{abstract}
OBJECTIVE Generalized-onset seizures are usually conceptualized as engaging bilaterally distributed networks with no clear focus. However, the authors previously reported a case series demonstrating that in some patients with generalized-onset seizures, focal seizure onset could be discovered after corpus callosotomy. The corpus callosum is considered to be a major pathway for seizure generalization in this group of patients. The authors hypothesized that, in patients with generalized-onset seizures, the structure of the corpus callosum could be different between patients who have lateralized seizures and those who have nonlateralized seizures after corpus callosotomy. The authors aimed to evaluate the structural difference through statistical analysis of diffusion tensor imaging (DTI) scalars between these two groups of patients.

METHODS Thirty-two patients diagnosed with generalized-onset motor seizures and without an MRI lesion were included in this study. Among them, 16 patients developed lateralized epileptic activities after corpus callosotomy, and the remaining 16 patients continued to have nonlateralized seizures after corpus callosotomy. Presurgical DTI studies were acquired to quantify the structural integrity of the corpus callosum.
\end{abstract}

RESULTS The DTI analysis showed significant reduction of fractional anisotropy (FA) and increase in radial diffusivity (RD) in the body of the corpus callosum in the lateralized group compared with the nonlateralized group.

CONCLUSIONS The authors' findings indicate the existence of different configurations of bilateral epileptic networks in generalized epilepsy. Generalized seizures with focal onset relying on rapid spread through the corpus callosum might cause more structural damage related to demyelination in the corpus callosum, showing reduced FA and increased RD. This study suggests that presurgical DTI analysis of the corpus callosum might predict the seizure lateralization after corpus callosotomy.

https://thejns.org/doi/abs/10.3171/2020.1.FOCUS19791

KEYWORDS diffusion tensor imaging; corpus callosotomy; generalized epilepsy; seizure lateralization; epileptic networks

$\mathrm{G}$ ENERALIZED-ONSET seizures are usually conceptualized as originating at some point within, and rapidly engaging, bilaterally distributed networks with no clear focus. ${ }^{5}$ The corpus callosum, the largest commissure connecting the two cerebral hemispheres, is conventionally considered to be responsible for bilateral synchronization in generalized epilepsy. ${ }^{20}$ Corpus callosotomy is a widely accepted and effective palliative proce- dure to mitigate drop attacks caused by bilateral synchronous epileptic discharges in patients with drug-resistant generalized epilepsy. ${ }^{12}$

However, the corpus callosum could also contribute to rapid spread of seizure activities from one hemisphere to the other hemisphere, causing seizure generalization..$^{22}$ Functional connectivity analysis further demonstrated that the corpus callosum is the major pathway for seizure gen-

ABBREVIATIONS $A D=$ axial diffusivity; $A U C=$ area under the ROC curve; $D T I=$ diffusion tensor imaging; $F A=$ fractional anisotropy; FMRIB = Functional MRI of the Brain; $\mathrm{MD}=$ mean diffusivity; $\mathrm{MNI}=$ Montreal Neurological Institute; $\mathrm{ROC}=$ receiver operating characteristic; $\mathrm{ROI}=$ region of interest; $\mathrm{RD}=$ radial diffusivity; $\mathrm{TBSS}=$ tract-based spatial statistics.

SUBMITTED November 4, 2019. ACCEPTED January 24, 2020.

INCLUDE WHEN CITING DOI: 10.3171/2020.1.FOCUS19791. 
eralization..$^{3,14}$ In some patients with generalized seizures, focal seizure onset might be discovered following disruption of the rapid propagation between two hemispheres after corpus callosotomy. ${ }^{2}$ Therefore, the bilateral epileptic networks in generalized epilepsy with focal onset could have greater reliance on the corpus callosum for seizure propagation than the bilateral epileptic networks in generalized epilepsy with nonfocal bilateral synchronization.

Alteration of structural integrity in the corpus callosum due to seizures often can be quantified through the analysis of diffusion tensor imaging (DTI). Abnormal fractional anisotropy (FA) and mean diffusivity (MD) in the corpus callosum have been observed in frontal lobe epilepsy, ${ }^{21}$ temporal lobe epilepsy, ${ }^{1}$ and generalized epilepsy. ${ }^{6,11}$

Generalized epilepsy with focal onset could heavily rely on the corpus callosum for seizure generalization, which could cause more damage to the structural integrity of the corpus callosum. In this study, we hypothesized that the structural integrity of the corpus callosum in patients with lateralized seizures after corpus callosotomy could be different from that in patients with nonlateralized seizures after corpus callosotomy. We aimed to evaluate the structural difference through statistical analysis of DTI scalars between these two groups of patients.

\section{Methods \\ Patients}

We retrospectively reviewed 105 patients with drugresistant epilepsy who underwent corpus callosotomy at Florida Hospital, AdventHealth in Orlando, Florida, between 2011 and 2016. The AdventHealth (Florida Hospital) IRB board approved the study. Thirty-two patients diagnosed with generalized-onset motor seizures and without an MRI lesion were included in this study. The presurgical comprehensive evaluation included 3T MRI, FDG-PET, prolonged video-EEG motoring, and ictal and interictal SPECT. ${ }^{16}$ All cases were defined as nonlesional due to the absence of focal or diffuse malformations, including cortical dysplasia, ischemic injury, hemorrhage, cerebral atrophy, sign of inflection, tuberous sclerosis, and dysgenesis, in the corpus callosum on MRI.

Either complete corpus callosotomy or an anterior two-thirds corpus callosotomy was performed based on the individualized clinical scenario of each patient. Intraoperative and postoperative MRI scans were acquired to confirm the proper execution of complete or anterior two-thirds corpus callosotomy as planned. If incomplete disconnection was identified, additional surgery was performed until the MRI sequences confirmed satisfactory disconnection. ${ }^{7}$ After corpus callosotomy, patients were reevaluated to observe alterations in their seizures. Based on post-corpus callosotomy scalp or intracranial EEG evaluation, patients were then divided into the seizure lateralized group or the seizure nonlateralized group.

\section{DTI Protocol}

Presurgical DTI images were acquired using a Signa HDxt 3T scanner (GE Healthcare) with an 8-channel phased array head coil following a protocol of TE 85.4 msec and TR 10,000 msec. A DTI diffusion axial scheme was used with 25 diffusion sampling directions. The bvalue was $1000 \mathrm{sec} / \mathrm{mm}^{2}$. The in-plane resolution was $2 \times$ $2 \mathrm{~mm}$, and the slice thickness was $4 \mathrm{~mm}$.

\section{Imaging Processing and Analysis}

The processing of DTI data utilized the FSL software package developed by the Oxford Centre for Functional MRI of the Brain (FMRIB). ${ }^{18}$ In short, DTI data were first processed by using FMRIB's Diffusion Toolbox in FSL to create fractional anisotropy (FA), mean diffusivity (MD), axial diffusivity (AD), and radial diffusivity (RD) maps. The Brain Extraction Tool was then applied to extract brain tissue from the maps. The created diffusion maps were aligned into a $1 \times 1 \times 1-\mathrm{mm}$ standard Montreal Neurological Institute (MNI) space using FMRIB's Nonlinear Registration Tool. Voxel-wise statistical analysis of the diffusion maps was carried out using the method of tractbased spatial statistics (TBSS). ${ }^{17}$ A mean FA image of all aligned FA images was calculated and thinned to create a mean FA skeleton representing the center of all tracts common to the group. Each subject's aligned diffusion maps were then projected onto this representative skeleton for voxel-wise statistical analysis between groups. MD, $\mathrm{AD}$, and RD data were processed in the same fashion and projected on the representative FA skeleton. The region of interest (ROI) was then segmented from the MNI space to create a binary mask for extracting the diffusion data within the corpus callosum.

\section{Statistical Analysis}

Voxel-wise statistical differences between the two groups of skeletonized DTI maps were analyzed by an unpaired t-test through nonparametric permutation-based inference with 5000 permutation using the randomize tool in FSL. Age, sex, age at seizure onset, seizure frequency, and duration of seizure history were included as covariates. The false positive caused by multiple comparison was corrected by threshold-free cluster enhancement. ${ }^{23}$ The statistical threshold for significance was set at $\mathrm{p}<$ 0.05 after correction.

The group differences in various clinical parameters were assessed using the nonparametric Mann-Whitney U-test. To explore the capability of using the DTI scalars for separating the lateralized group and the nonlateralized group on an individual level, receiver operating characteristic (ROC) curve analysis was applied through the calculation of a true-positive rate (sensitivity) versus false-positive rate (1 - specificity) at varied cutoff thresholds. The area under the curve (AUC) was calculated to determine the performance of each classifier. The statistical analyses were carried out using SigmaPlot.

\section{Results \\ Clinical Profile}

Among 32 patients, 16 developed lateralized epileptic activities after corpus callosotomy, and the remaining 16 patients continued having nonlateralized seizures. In both groups, 14 patients underwent complete corpus callostomy and 2 underwent an anterior two-thirds corpus callostomy. In the lateralized group, 7 patients' seizures lateralized to 
TABLE 1. Clinical descriptions of each group

\begin{tabular}{lccc}
\hline & Lateralized & Nonlateralized & $p$ Value* \\
\hline No. of patients & 16 & 16 & \\
\hline Sex & 10 & 10 & \\
\hline$\quad$ Male & 6 & 6 & \\
\hline Female & $9.37 \pm 8.22$ & $9.31 \pm 6.33$ & 0.72 \\
\hline Mean age, yrs & $3.18 \pm 4.92$ & $2.94 \pm 3.87$ & 0.42 \\
\hline $\begin{array}{l}\text { Mean age at seizure onset, } \\
\text { yrs }\end{array}$ & $3.26 \pm 3.77$ & $1.83 \pm 1.82$ & 0.61 \\
$\begin{array}{l}\text { Mean seizure frequency } \\
\text { per day }\end{array}$ & & \\
\hline $\begin{array}{l}\text { Mean seizure history, yrs } \\
\text { Mean values are presented as mean } \pm \text { SD. }\end{array}$ & & \\
* Calculated using nonparametric Mann-Whitney & U-test. & \\
\end{tabular}

the right side and in the remaining 9 patients, they lateralized to the left side. Six patients' seizures were not further localized, 5 patients' seizures involved the parietal lobes, 4 involved the frontal lobes, and 2 involved the temporal lobes. Seven patients in the lateralized group later underwent resective surgical intervention after corpus callosotomy. Table 1 lists the clinical descriptions of each group and their differences. There was no significant difference between these two groups of patients based on the listed parameters. The 1-year postsurgery seizure outcomes according to the ILAE (International League Against Epilepsy) classification are shown in Table 2 . Among 7 patients in the lateralized group who later underwent resective surgeries, 2 had class I, 1 had class III, and 2 had class $\mathrm{V}$ outcomes, and 2 patients were lost follow-up. Overall, the lateralized group showed better surgical outcomes over the nonlateralized group.

\section{Correlation Between White Matter Integrality and Other Confounding Factors}

We evaluated other factors that might affect DTI maps, such as sex, age, age at seizure onset, seizure frequency, and the length of seizure history. In these 32 patients, the results showed that age had a significant positive effect on FA and significant negative effect on $\mathrm{MD}, \mathrm{AD}$, and RD throughout most regions of the corpus callosum. The length of seizure history did not show a significant effect after adjusting for age. Sex, age at seizure onset, and seizure frequency also did not show significant effects.

\section{DTI Analysis in Relation to Seizure Lateralization}

When comparing the difference between the lateralized and the nonlateralized groups, significant FA reduction was found in the body of the corpus callosum in the lateralized group. The body of the corpus callosum also demonstrated a significantly higher RD value in the lateralized group than in the nonlateralized group. MD in the body of the corpus callosum also was higher in the lateralized group, but the results were not significant. Figure 1 illustrates the results of TBSS analysis of FA and RD.

The region in the body of the corpus callosum that
TABLE 2. Seizure outcomes

\begin{tabular}{lccccccc}
\hline & \multicolumn{7}{c}{ ILAE Class } \\
\cline { 2 - 8 } & I & II & III & IV & V & IV & NA \\
\hline Lateralized & 6 & 0 & 1 & 1 & 3 & 0 & 5 \\
\hline Nonlateralized & 0 & 0 & 3 & 6 & 1 & 1 & 5 \\
\hline
\end{tabular}

NA = not applicable, lost to follow-up.

Values are number of patients.

showed significant differences in group-level analysis was identified as an ROI. The mean values of FA and RD in the ROI were calculated for each patient as potential classifiers. Figure 2 illustrates the differences of mean FA and mean RD between the two groups. The differences were significant ( $p<0.05$, Mann-Whitney U-test) for both mean FA and RD. Figure 3 depicts the ROC analysis of using mean FA and mean RD for individual-level classification. At the cutoff threshold of $<0.5306$, the mean FA achieved a sensitivity of 0.790 and a specificity of 0.760 . At the cutoff threshold of $>0.000722$, the mean RD achieved 0.842 sensitivity and 0.706 specificity. The AUC values of using the mean FA and mean RD as classifiers were $0.848(95 \%$ CI $0.721-0.975, \mathrm{p}<0.001)$ and $0.842(0.714-0.970, \mathrm{p}<$ 0.001 ), respectively. The AUC values suggested that the mean FA and mean RD of the ROI can perform a good classification between these two groups of patients.

\section{Discussion}

Our previous study demonstrated that some generalized seizures could be induced by focal onset with rapid spread through the corpus callosum. ${ }^{2}$ Under this circumstance, the corpus callosum is considered to be a major pathway of seizure propagation in the bilateral epileptic network, which might have different configurations from nonfocal bilateral synchronization. ${ }^{3}$ The results of DTI analysis in this study demonstrated that the corpus callosum suffered more structural damage related to demyelination in the lateralized group than in the nonlateralized group, causing reduced FA and increased RD. ${ }^{4}$ This finding further indicates that different configurations of bilateral epileptic networks exist in generalized epilepsy with greater or less reliance on the corpus callosum, which inflicts varied structural alterations along their respective pathways.

This study suggests that presurgical DTI analysis of the corpus callosum might predict the seizure lateralization after corpus callosotomy. The lateralized group showed overall better surgical outcomes, even after excluding the patients with subsequent resective surgeries. Therefore, the proposed preoperative DTI analysis of the corpus callosum might provide an additional prognostic value on evaluating the risks and benefits of corpus callosotomy before the operation. However, the results were produced by a highly selected group of young patients with negative MRI results. This cohort does not reflect a real clinical population undergoing corpus callosotomy. We decided to start the investigation with this group of patients to eliminate possible variability caused by different types of pathologies and potential coregistration errors because of 
(A) FA

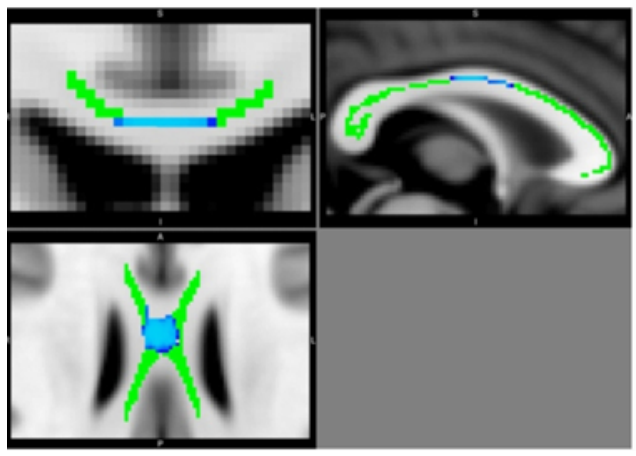

(B) RD

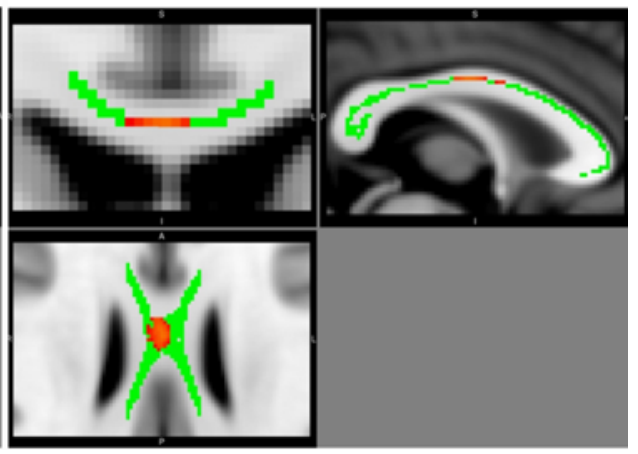

FIG. 1. TBSS analysis of FA and RD. The green skeleton represents the center of the white matter tracts of the corpus callosum used in the analysis overlaid on the MNI standard brain. A: The blue pixels indicate the area with significant reduction of FA in the lateralized group compared with the nonlateralized group ( $p<0.05$ corrected). B: The red pixels indicate the area with a significant increase in RD in the lateralized group compared with the nonlateralized group ( $p<0.05$ corrected).

dysmorphic anatomy. Other researchers have reported a good chance of $44 \%$ to $50 \%$ to develop seizure localization after corpus callosotomy in their patient groups..$^{10,19}$ Further investigations are warranted to determine if the proposed methodology can be applied to a more diverse group of patients and whether seizure lateralization after corpus callosotomy could significantly improve overall outcomes.

In this study, the regions with significantly reduced FA and increased RD were in the body of the corpus callosum. We suspect that the focality could be related to the location of lateralized epileptic activities after corpus callosotomy. The majority of patients in the lateralized group had focal epileptic activities in the frontal and parietal regions involving the motor and sensory cortices, which are highly connected along the body of the corpus callosum through callosal fibers. ${ }^{13}$ It is possible that, with different focal seizure onset locations, the region of impact within the corpus callosum could also be shifted based on onset location. Instead of using a fixed ROI for every patient, a more sophisticated classification methodology using feature and pattern recognition on the individual level through a machine learning technique can be developed. This approach will further increase the diagnostic classification accuracy. However, it will require a larger sample size with different seizure localizations to confirm this assumption further.

Regarding other confounding factors that might affect diffusion tensor parameters, age was found to have a significant impact in this group of young patients. A largescale study also reported that age showed a positive correlation with FA and a negative correlation with MD in the corpus callosum at early ages. ${ }^{9}$ Nonetheless, there is no good agreement about how other clinical seizure factors might be associated with diffusion parameters. Some studies have reported that reduced FA in the corpus callosum was related to age at seizure onset and seizure frequency in patients with temporal lobe epilepsy and juvenile myoclonic epilepsy, respectively. ${ }^{8,15}$ This study and other studies found no correlation in generalized epilepsy and frontal lobe epilepsy. ${ }^{6,21}$ Because each study had a unique patient population, the underlying causes that produced discrepancies among different studies were unclear.

\section{Conclusions}

This study provides new insight about seizure lateralization after corpus callosotomy in patients with generalized epilepsy. DTI analysis demonstrates its potential ability to understand the different configurations of bilateral epileptic networks in generalized epilepsy.
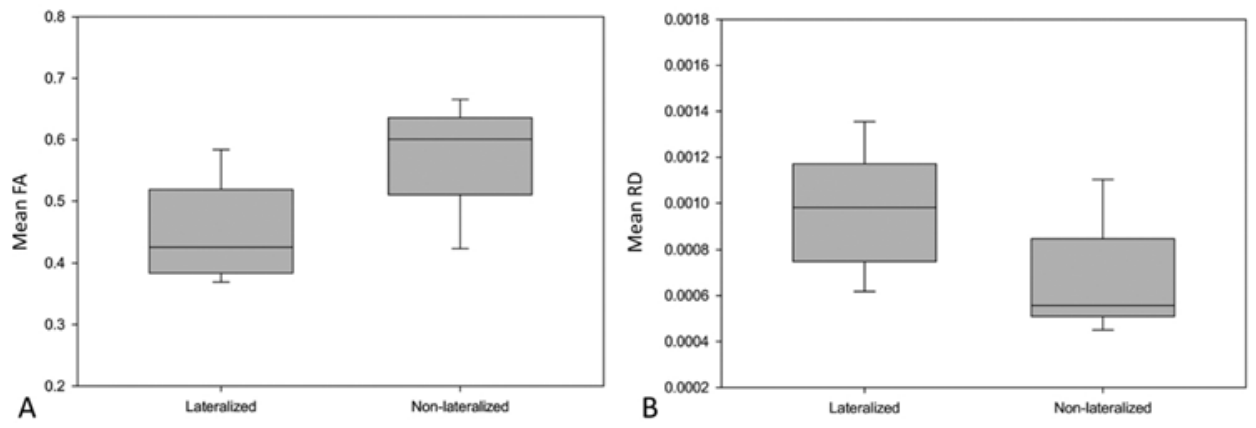

FIG. 2. Box plots of mean FA (A) and mean RD (B) of the ROI in the lateralized group versus the nonlateralized group. The horizontal line represents the median, the error bars indicate 10th and 90th percentiles, and the upper limit and lower limit of the box indicate 25 th and 75 th percentiles. 

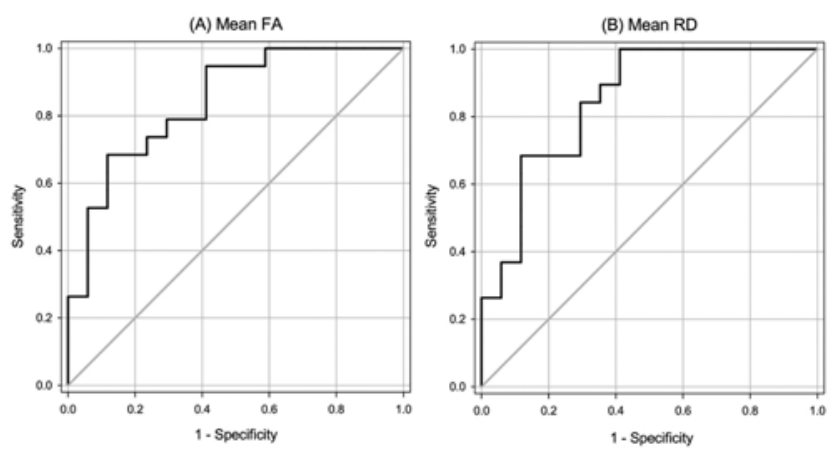

FIG. 3. ROC curves of the mean $F A(A)$ and the mean $R D(B)$.

\section{References}

1. Caligiuri ME, Labate A, Cherubini A, Mumoli L, Ferlazzo $\mathrm{E}$, Aguglia U, et al: Integrity of the corpus callosum in patients with benign temporal lobe epilepsy. Epilepsia 57:590596, 2016

2. Chen PC, Baumgartner J, Seo JH, Korostenskaja M, Lee KH: Bilateral intracranial EEG with corpus callosotomy may uncover seizure focus in nonlocalizing focal epilepsy. Seizure 24:63-69, 2015

3. Chen PC, Castillo EM, Baumgartner J, Seo JH, Korostenskaja M, Lee KH: Identification of focal epileptogenic networks in generalized epilepsy using brain functional connectivity analysis of bilateral intracranial EEG signals. Brain Topogr 29:728-737, 2016

4. Feldman HM, Yeatman JD, Lee ES, Barde LH, Gaman-Bean S: Diffusion tensor imaging: a review for pediatric researchers and clinicians. J Dev Behav Pediatr 31:346-356, 2010

5. Fisher RS, Cross JH, D'Souza C, French JA, Haut SR, Higurashi N, et al: Instruction manual for the ILAE 2017 operational classification of seizure types. Epilepsia 58:531-542, 2017

6. Focke NK, Diederich C, Helms G, Nitsche MA, Lerche H, Paulus W: Idiopathic-generalized epilepsy shows profound white matter diffusion-tensor imaging alterations. Hum Brain Mapp 35:3332-3342, 2014

7. Kim GH, Seo JH, Schroff S, Chen PC, Lee KH, Baumgartner J: Impact of intraoperative 3-T MRI with diffusion tensor imaging on hemispherectomy. J Neurosurg Pediatr 19:6369, 2017

8. Kim JH, Suh SI, Park SY, Seo WK, Koh I, Koh SB, et al: Microstructural white matter abnormality and frontal cognitive dysfunctions in juvenile myoclonic epilepsy. Epilepsia 53:1371-1378, 2012

9. Lebel C, Beaulieu C: Longitudinal development of human brain wiring continues from childhood into adulthood. J Neurosci 31:10937-10947, 2011

10. Lin JS, Lew SM, Marcuccilli CJ, Mueller WM, Matthews AE, Koop JI, et al: Corpus callosotomy in multistage epilepsy surgery in the pediatric population. J Neurosurg Pediatr 7:189-200, 2011

11. Liu M, Concha L, Beaulieu C, Gross DW: Distinct white matter abnormalities in different idiopathic generalized epilepsy syndromes. Epilepsia 52:2267-2275, 2011

12. Malmgren K, Rydenhag B, Hallböök T: Reappraisal of corpus callosotomy. Curr Opin Neurol 28:175-181, 2015
13. Park HJ, Kim JJ, Lee SK, Seok JH, Chun J, Kim DI, et al: Corpus callosal connection mapping using cortical gray matter parcellation and DT-MRI. Hum Brain Mapp 29:503516, 2008

14. Peng SJ, Hsin YL: Functional connectivity of the corpus callosum in epilepsy patients with secondarily generalized seizures. Front Neurol 8:446, 2017

15. Riley JD, Franklin DL, Choi V, Kim RC, Binder DK, Cramer SC, et al: Altered white matter integrity in temporal lobe epilepsy: association with cognitive and clinical profiles. Epilepsia 51:536-545, 2010

16. Seo JH, Holland K, Rose D, Rozhkov L, Fujiwara H, Byars A, et al: Multimodality imaging in the surgical treatment of children with nonlesional epilepsy. Neurology 76:41-48, 2011

17. Smith SM, Jenkinson M, Johansen-Berg H, Rueckert D, Nichols TE, Mackay CE, et al: Tract-based spatial statistics: voxelwise analysis of multi-subject diffusion data. Neuroimage $31: 1487-1505,2006$

18. Smith SM, Jenkinson M, Woolrich MW, Beckmann CF, Behrens $\mathrm{TE}$, Johansen-Berg $\mathrm{H}$, et al: Advances in functional and structural MR image analysis and implementation as FSL. Neuroimage 23 (Suppl 1):S208-S219, 2004

19. Taraschenko O, Pedavally S, Samson KK, Puccioni MJ, Madhavan D: Anterior corpus callosotomy in patients with drug-resistant epilepsy: invasive EEG findings and seizure outcomes. Epilepsy Behav Case Rep 9:12-18, 2017

20. Tukel K, Jasper H: The electroencephalogram in parasagittal lesions. Electroencephalogr Clin Neurophysiol 4:481-494, 1952

21. Widjaja E, Kis A, Go C, Snead OC III, Smith ML: Bilateral white matter abnormality in children with frontal lobe epilepsy. Epilepsy Res 108:289-294, 2014

22. Wieshmann UC, Milinis K, Paniker J, Das K, Jenkinson $\mathrm{MD}$, Brodbelt A, et al: The role of the corpus callosum in seizure spread: MRI lesion mapping in oligodendrogliomas. Epilepsy Res 109:126-133, 2015

23. Winkler AM, Ridgway GR, Webster MA, Smith SM, Nichols TE: Permutation inference for the general linear model. Neuroimage 92:381-397, 2014

\section{Disclosures}

Dr. Baumgartner: consultant for PMT Corp. and Monteris Medical.

\section{Author Contributions}

Conception and design: Chen, Messina, Castillo. Acquisition of data: Chen, Baumgartner, Seo. Analysis and interpretation of data: Chen, Messina. Drafting the article: Chen, Messina. Critically revising the article: all authors. Reviewed submitted version of manuscript: all authors. Approved the final version of the manuscript on behalf of all authors: Chen. Statistical analysis: Chen. Administrative/technical/material support: Chen, Messina, Castillo. Study supervision: Chen, Castillo, Lee.

\section{Correspondence}

Po Ching Chen: Epilepsy Center, Neuroscience Institute, Florida Hospital, AdventHealth, Orlando, FL.po-ching.chen@ adventhealth.com. 